\title{
DETECTION OF ORIENTED NUMBER PLATE IN VEHICLE USING AUTOCORRECTION FEATURE FROM GRAY LEVEL CO-OCCURENCE MATRIX
}

\author{
Veena M.N ${ }^{1}$, Shruthi S.J ${ }^{2}$ and Vasudev.T ${ }^{2}$ \\ ${ }^{1}$ P.E.T Research Foundation, \\ P.E.S. College of Engineering, Mandya, Karnataka, India \\ veenadisha1@gmail. com \\ ${ }^{2}$ Maharaja Institute of Technology, Mysore, Karnataka, India \\ shruthijayaram131@gmail.com, vasu@mitmysore.in
}

\begin{abstract}
The efficiency of an automatic number plate recognition system depends directly on the proper effective preprocessing of the number plate. The OCRs available for recognition are capable of reading the number plates which are in proper orientation of 00. In many situations the vehicle number plates captured may be in any different orientation like 900, 1800 and 2700. These orientations in number plates are due to declamping of number plate at one end or toppling of vehicle. Such differently oriented number plates cannot be subjected for reading by OCRs and such situations require the system to detect the direction of orientation and correct the same before subjecting the same for reading. This paper proposes a work to detect the orientation of segmented number plates from vehicle image using autocorrelation feature from gray level cooccurrence matrix. A good volume of training samples are generated synthetically to train the system and the system is tested using sufficient test samples. The results of system shows an overall efficiency of $65.61 \%$ and performs an essential preprocessing in an automatic number plate recognition system.
\end{abstract}

\section{KEYWORDS}

Number Plate, Orientation, Autocorrelation, OCRs

\section{INTRODUCTION}

Vehicle Number Plate Recognition(VNPR) system is an image processing application, developed to track the information about vehicles through the number plates. This application is gaining popularity in security and traffic monitoring systems. The number plate recognition system is important for variety of applications like automatic traffic congestion charge system, access control, tracing of stolen vehicles and identification of vehicles for traffic rules violations. The VNPR system plays a major role in automatic monitoring of traffic rules and maintains law enforcement on public roads. A VNPR system goes through a series of preprocessing task for

David C. Wyld et al. (Eds) : CSEN, AISO, NCWC, SIPR - 2015

pp. 21-27, 2015. ( ) CS \& IT-CSCP 2015

DOI : $10.5121 /$ csit.2015.51403 
efficient automatic reading. The generic OCRs available are designed to read the text in $0^{0}$ orientations and are not capable of reading text in other orientations. The number plates segmented from captured vehicle images may not be in correct orientation i.e number plate instead of being at $0^{\circ}$ orientation, it may be in any one of the $90^{\circ}, 180^{\circ}$ and $270^{\circ}$ orientations. The samples of segmented number plate in different orientation directions are shown in Figure1.

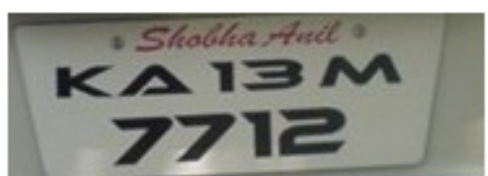

Figure. 1 (a) $0^{0}$ Orientation

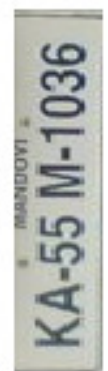

(b) $90^{\circ}$ Orientation

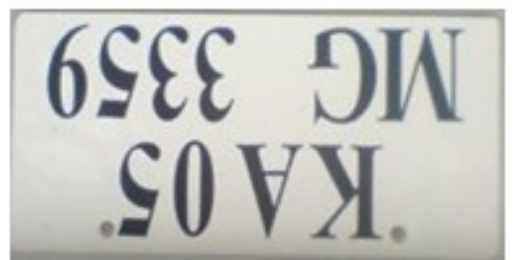

(c) $180^{\circ}$ Orientation

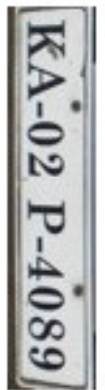

(d) $270^{\circ}$ Orientation

The different directions of orientations in number plates are due to unclamped number plates at one end or it may be due to toppled vehicles. Since OCRs do not to read such number plate oriented in other directions, the automatic number plate recognition (ANPR) system demands requirement of a preprocessing stage to detect the orientation of number plate and correct the same before subjecting for reading by OCRs. This requirement has motivated us to explore a method to detect the direction of orientation of the plate in vehicle images.

The work presented in this paper focuses on implementation of orientation detection in vehicle number plate. The rest of the paper is organized as follows section 2 gives brief survey about related research work. Section 3 presents the model designed for detection of orientation in vehicle number plate. Section 4 discuss about the experimental results and a brief conclusion is provided in section 5 .

\section{LITERATURE SURVEY}

Many researchers have proposed good number of methods to detect tilt in the number plate of vehicles[1]. Tilt is the angular rotation noticed in the number plate. These methods are designed to detect the tilt which are less than $35^{\circ}$. These approaches are based on Principle Component Analysis [2], Fourier Transform[3], Hough Transform[4], Nearest neighbor connectors[5], Extreme points[6] and Moments[7,8]. But most approaches fail to detect the orientations of $90^{\circ}$, $180^{\circ}$ and $270^{\circ}$. In literature sufficient quantum of works are reported on text skew detection in documents. The work in [9] proposed a modified approach of Hough transform for skew detection and correction of document images, the algorithm is computationally less efficient. The work in [10] proposed an integrated skew detection and correction using Fourier transform and DCT which is also computationally less efficient. Many different methodologies to detect skew in a given document page [11] were discussed. A traditional projection profile approach is proposed in [12]. In this approach features are extracted from each projection profile to determine the skew angle and it is quite cost effective. The improved nearest-neighbor based document skew detection method is proposed in [13] to estimate skew in documents respective to skew angle limitation. Another approach uses k-NN [14] clustering of the connected components. This approach has a relatively high accuracy but has a large computational cost, independent of the detection range. A skew detection method using the cross correlation between the text lines at a 
fixed distance [15] is based on the fact that the correlation between vertical lines in an image is maximum for a skewed document, is presented. It is found that the proposed method is computationally expensive and gives lesser accuracy. A bottom up technique for skew estimation based on nearest neighbor clustering is proposed in [16]. In this method, nearest neighbors of all the connected components are determined. Since only one nearest neighbor connectivity is made for each component, connection with noisy sub parts of characters would reduce the accuracy of the method. The above reported techniques have some limitations and depends on the factors like, speed, suitable only for text of sufficient size. Few techniques provide accurate results but slow in processing and other few techniques are cost effective but efficient in speed and accuracy. Some works are reported in literature to recognize the number plates in vehicle using size features of number plates[17]. But these approaches fail in distinguishing between number plate in $0^{\circ}$ and $180^{\circ}$ as well as between $90^{\circ}$ and $270^{\circ}$ for rectangle shaped number plates. In addition, the methods cannot be extended for square shaped number plates.

To the best of our knowledge, we could find a couple of works on detection of orientation in document images [18] and [19]. These methods are suitable for large text document and not ideal for number plate which contain very little text or very few characters. Hence a more suitable method is required to detect orientation of number plates and the work presents an attempt made in this direction.

\section{PROPOSED MODEL}

Input to the system is assumed to be the segmented number plate from the vehicle image. The sequence of stages in the work is shown in Figure.2. The process begins with necessary preprocessing to enhance the input number plate image for better character segmentation and character recognition. The preprocessed input image is subjected to detect the direction of orientation using autocorrelation feature from gray level co occurrence matrix (GLCM). Once the direction of the orientation is detected, next the same is corrected by rotating the segmented number plate in the counter direction. The subsequent subsections discuss the process in detail.

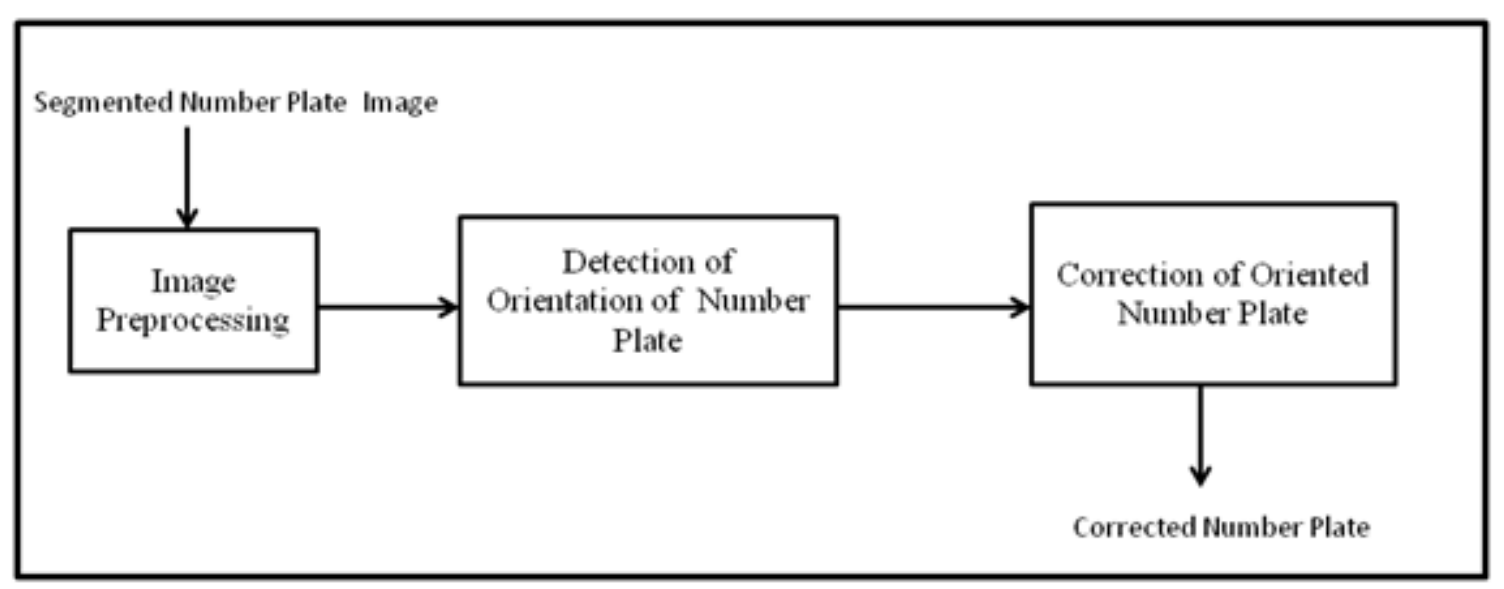

Figure 2. Stages in the Proposed Model 


\subsection{Preprocessing}

The input image is converted into a grayscale image for easy analysis as it consists of only two color channels. The preliminarily preprocessing is carried out to remove noise using median filtering [20] from the input image. Median filter is a non-linear filter, which replaces the gray value of a pixel by the median of the gray values of its neighbours. A $3 \times 3$ mask is used to get eight neighbours of a pixel and their corresponding gray values. The gray value of the centre pixel of the mask is replaced by the median of the gray values of the pixels within the mask. This operation removes salt-and-pepper noise from the image. Figure.3 (a), (b) and (c) show the input, gray scale converted and filtered images respectively.

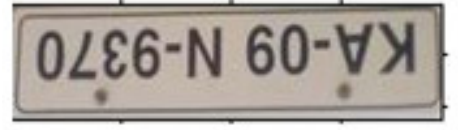

Figure 3. (a). Input Image

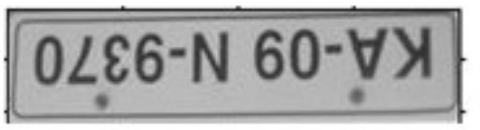

(b). Gray Scale Image

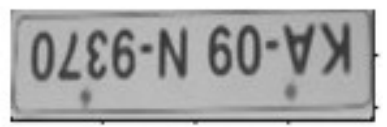

(c). Median Filtered Image

\subsection{Orientation detection using Gray Level Co-variance Matrix (GLCM)}

Texture is an important feature of an image. Texture plays an important role in many machine vision tasks such as surface inspection, scene classification, surface orientation and shape determination. Texture is characterized by the spatial distribution of gray levels in a neighbourhood. We can define texture as repeating patterns of local variations in image intensity which are to fine to be distinguished as separate objects at the observed resolution. Thus, a connected set of pixels satisfying a given gray-level property which occur repeatedly in an image region constitutes a textured region. To describe the texture of the region three approach are used in image processing these are statistical, structural and spectral. Statistical approaches specify the characterization of the textures by smooth, coarse, grainy, silky and so on. Since the texture is a spatial property, a simple one dimensional histogram is not useful in characterizing texture. In order to capture the spatial dependence of gray level values which contribute to the perception of texture, a two-dimensional dependence matrix known as gray level co occurrence matrix [21] is extensively used in texture analysis. Using a statistical approach such as co-occurrence matrix will help to provide valuable information about the relative position of the neighbouring pixels in an image. Another measure that has been used extensively in GLCM is the autocorrelation function[22] and this feature is mainly used for detection of orientation patterns in images.

\subsubsection{Autocorrelation}

The autocorrelation function [21] $\mathrm{P}(\mathrm{K}, \mathrm{L})$ for an image ' $\mathrm{f}$ ' is given by equation (1)

$$
P(K, L)=\frac{\frac{1}{(N-K)(N-L)} \sum_{i=1}^{(N-K)} \sum_{j=1}^{(N-1)} f(i, j) f(i+k, j+l)}{1 / N 2 \sum_{i=1}^{N} \sum_{j=1}^{N} f 2(i, j)} \quad 0 \leq K, l
$$

For images comprising repetitive texture pattern the autocorrelation function exhibits periodic behavior with a period equal to the spacing between adjacent texture primitives. When the texture is coarse, the autocorrelation function drop off slowly, where as for fine texture it drops off rapidly. The measure of periodicity of texture as well as a measure of the scale of the texture 
primitives. The autocorrelation range values for different orientations are computed and tabulated from the training samples. The Table.1 represents the Autocorrelation value ranges obtained from training samples for different orientations with limited overlaps.

Table 1 Autocorrelation value ranges

\begin{tabular}{|c|c|}
\hline Orientation & Autocorrelation \\
\hline $0^{\circ}$ & $>50$ \\
\hline $90^{\circ}$ & $12-31$ \\
\hline $180^{0}$ & $45-50$ \\
\hline $270^{\circ}$ & $32-44$ \\
\hline
\end{tabular}

\section{EXPERIMENTAL RESULTS}

The experiments are conducted through testing 495 segmented vehicle number plate images obtained in different orientations. For each test sample the autocorrelation value is computed and the classification is made based on range values specified in Table 1. The Figures 4 (a), (b), (c),(d), (e), (f) and (g) shows few samples of oriented input images and corresponding corrected number plates.

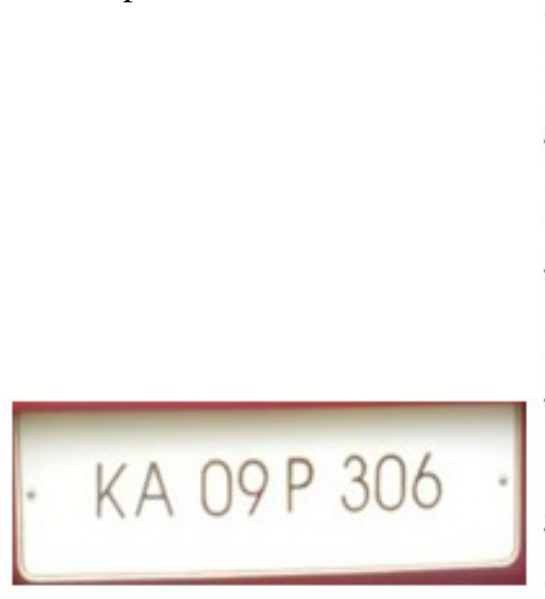

Figure 4. (a) $0^{0}$ Orientation

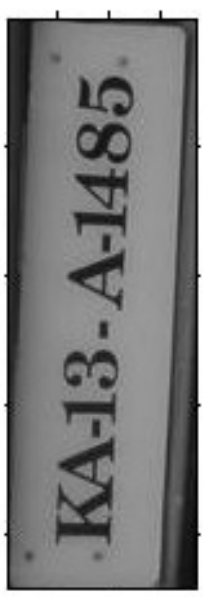

(b). $90^{\circ}$ Orientation
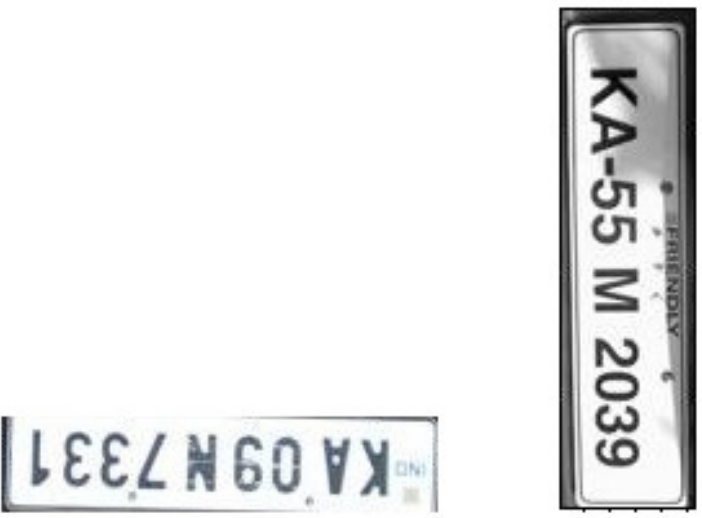

(c). $180^{\circ}$ Orientation

(d). $270^{0}$ Orientation

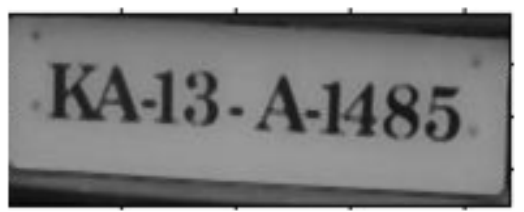

(e). Corrected image of (b)

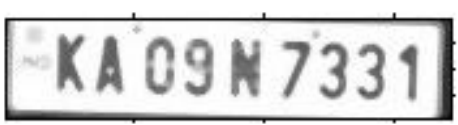

(f). Corrected image of (c)

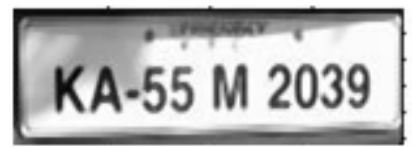

(g). Corrected image of (d)

The results of the experiments are tabulated in table 2 . The result of overall efficiency of proposed method is $65.61 \%$ with $22.16 \%$ of wrong detections and $11.85 \%$ of rejections. The rejections are due to failures in autocorrelation threshold values. The wrong detections are due to overlapping of autocorrelation threshold values. 
Table 2 Results of Detection Orientation in Vehicle Number Plate Image

\begin{tabular}{|c|l|l|l|l|}
\hline Orientation & $\begin{array}{l}\text { No. of } \\
\text { Samples }\end{array}$ & $\begin{array}{l}\text { Correct } \\
\text { Detections }\end{array}$ & $\begin{array}{l}\text { Wrong } \\
\text { Detections }\end{array}$ & Rejections \\
\hline $0^{0}$ & 100 & $75(75 \%)$ & $17(17 \%)$ & $8(8 \%)$ \\
\hline $90^{0}$ & 145 & $89(61.38 \%)$ & $36(24.82 \%)$ & $20(13.79 \%)$ \\
\hline $180^{0}$ & 115 & $70(60.87 \%)$ & $30(26.09 \%)$ & $15(13.04 \%)$ \\
\hline $270^{0}$ & 135 & $88(65.18 \%)$ & $28(20.74 \%)$ & $17(12.59 \%)$ \\
\hline Total & $\mathbf{4 9 5}$ & $\mathbf{3 2 2}(\mathbf{6 5 . 6 1 \%})$ & $\mathbf{1 1 1}(\mathbf{2 2 . 1 6 \% )}$ & $\mathbf{6 0 ( 1 1 . 8 5 \% )}$ \\
\hline
\end{tabular}

\section{CONCLUSION}

The work presented in this paper detects oriented number plates in vehicles which is an essential preprocessing required for an Vehicle Number Plate Recognition system under certain situations. The direction of orientation is detected using autocorrelation features from GLCM. The overall efficiency of proposed method is $65.61 \%$. Misclassification is more between $\left(0^{\circ}\right.$ and $\left.180^{\circ}\right)$ and $\left(90^{\circ}\right.$ and $\left.270^{\circ}\right)$ due to overlapping of autocorrelation threshold values and rejections are due to computed in autocorrelation values does not fit in threshold ranges. Since it is an initial attempt made in this direction the success rate is relatively less. However there is much scope to minimize the misclassification using multi features of GLCM with multilevel classification which is under investigation.

\section{REFERENCES}

[1] Muhammad Kamal Hossen and Kaushik Deb " Vehicle License Plate Detection and Tilt Correction Based on HSI Color Moel and SUSAN Corner Detector ", Smart Computing Review, Vol.4 No. 5, 2014.

[2] Rajesh Bodade, Ram Bilas Pachori, Aakash Gupta, Pritesh Kanani, Deepak Yadav, "A Novel Approach for Automated Skew Correction of Vehicle Number Plate Using Principal Component Analysis" 2012 IEEE International Conference on Computational Intelligence and Computing Research.

[3] Lowther, S., Chandhran, V., Sridharan, S. An accurate method for skew determination in document images. In Proceedings of the Digital Image Computing Techniques and Applications. Melbourne (Australia), 2002.

[4] Arulmozhi, Arumuga Perumal, Thanooja Priyadarsini, Krishnan Nallaperumal, "Image Refinement Using Skew Angle Detection and Correction for Indian License Plates" 2012 IEEE International Conference on Computational Intelligence and Computing Research.

[5] Y. Lu, C. L. Tan., Improved Nearest Neighbor Based Approach to Accurate Document Skew Estimation, Department of Computer Science, School of Computing, National University of Singapore, Kent Ridge Singapore,2003.

[6] Marian Wagdy, Ibrahima Faye, Dayang Rohaya. Document Image Skew Detection and Correction Method Based on Extreme Points. In: 2014 IEEE.

[7] K. George ,K. Nicholas., A Fast High Precision Algorithm for the Estimation of Skew Angle Using Moments, Department of Informatics and Telecommunications University of Athens,2002.

[8] B. Darko , R. M. Dragan, An Algorithm for the Estimation of the Initial Text Skew, University of Belgrade, Technical Faculty Bor, Mining and Metallurgy Institute, Department of Informatics, Serbia, 2012.

[9] Deepak kumar, Dalwinder singh "modified approach of Hough transform for skew detection and correction in documented image" IJRCS, in 2012. 
[10] Madir kaur, Simple Jindhal "an integrated skew detection and correction using fast fourier transform nd DCT",IJST,December 2013.

[11] R.singh and R kaur "skew detection in image processing”, IJCTA, issn 2229-6093, vol 4,no.3(2013),pp.478-485.Postl, W., (1986).

[12] Detection of linear oblique structures and skew scan in digitized documents. In: Proc. 8th Internat. Conf. on Pattern Recognition, Paris, France, 687-689.

[13] M. Sarfraz, S.A. Mahmoud and Z. Rasheed,On Skew Estimation and Correction of Text, Computer Graphics, Imaging and Visualisation (CGIV 2007) 0-7695-2928-3/07.

[14] G.S.Lehal and Renu Dhir, (1999) “A Range Free Skew Detection Technique for Digitized Gurmukhi Script Documents", Proceedings 5th International Conference of Document Analysis and Recognition,Banglore,pp.147-152.

[15] Yan, H., "Skew correction of document images using interline cross correlation", Computer Vision, Graphics, and Image Processing 55,1993, pp 538-543.

[16] Hashizume, P. S. Yeh, A. Rosenfeld, "A method of detecting the orientation of aligned components", Pattern Recognition Letters, 1996,pp. 125-13

[17] Ahmed, M.Sarfaz, A. Zidouri and K.G AI-Khatib, " License plate recognition system", In the 10th IEEE International Conference on Electronics, Circuits and Systems, pp.898-901 (2003).

[18] Vasudev .T et.al. " Language Independent Skew Detection and Correction of Printed Text Document Images : A Non-rotational Approach, July 2006, VIVEK: Journal of Artificial Intelliegence, Vol.16, No.10,pp.8-15,2006.

[19] Vasudev .T, et.al " Detection and Correction of Page Orientation in Monochrome Textual Document Image" Journal of Computer Science, Vol. 2, No.1,PP.465-473, Jan-2008.

[20] Gonzalez .R.C, R.E .Woods ,"Digital Image Processing", 2nd Edition, Printice Hall, Englewood Cliffs , NY 2002

[21] Andrik Rampun, Harry Strange, " Texture Segmentation using different orientations of GLCM features", MIRAGE'13, June 06-07-2013, Berlin, Germany.

[22] Ramesh Jain, Rangachar Kastrui " Machine Vision" Published by Mc.Graw-Hill, Inc, ISBN 0-070320187,1995 .

\section{AUTHORS}

Veena M.N, obtained her Master of Computer Applications(MCA) from University of Mysore in 1998. She received her one more Master degree in Computer science and technology from University of Mysore in 2007. She is having 15 years of experience in academics and her area of research is Digital Image Processing and Computer Vision. She is pursuing doctoral degree under University of Mysore.

Shruthi S.J, Obtained her Bachelor degree in Information Science and Engineering from Ghousia College of Engineering, Ramanagara in 2013. She is currently pursuing Master degree in Computer Science and Engineering under VTU, Belgaum.

Vasudev .T is Professor, in the Department of Master of Computer Applications, Maharaja Institute of Technology, Mysore. He obtained his Bachelor of Science and post graduate diploma in computer programming with two Masters Degrees one in Computer Applications and other one is Computer Science and Technology. He was awarded PhD. in Computer Science from University of Mysore. He is having 30 years of experience in academics and his area of research is Digital Image Processing specifically document image processing.
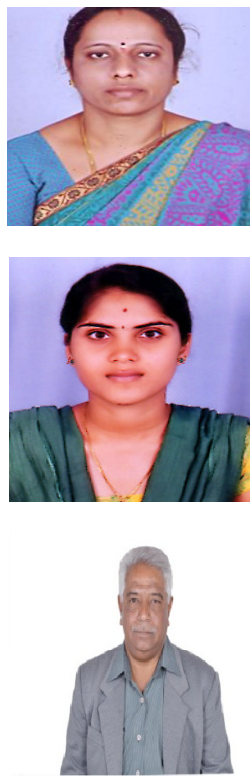